\title{
Is electronic monitoring of adherence to inhaled steroids useful in troublesome preschool wheeze?
}

\author{
Yvonne Bingham ${ }^{1}$, James Cook$^{1}$, Pippa Hall ${ }^{2}$, Angela Jamalzadeh ${ }^{2}$, Rachael \\ Moore-Crouch ${ }^{2}$, Andrew Bush ${ }^{3}$, Louise Fleming ${ }^{3}$, and Sejal Saglani ${ }^{1}$ \\ ${ }^{1}$ Imperial College London Faculty of Medicine - South Kensington Campus \\ ${ }^{2}$ Royal Brompton and Harefield NHS Foundation Trust \\ ${ }^{3}$ Imperial College and Royal Brompton Hospital, London, UK
}

May 19, 2020

\begin{abstract}
Background: Adherence to inhaled corticosteroids (ICS) is suboptimal in school-age children with asthma. Little is known about adherence to ICS in preschool children with troublesome wheeze. Children with aeroallergen sensitisation, or those with multiple trigger wheeze (MTW), should be differential ICS responders. We hypothesised that adherence to ICS and symptom control are only positively related in atopic children, or those with MTW. Methods: Patients aged 1-5 years with recurrent wheeze prescribed ICS were recruited from a tertiary respiratory clinic. Clinical phenotype and aeroallergen sensitisation were determined, and adherence assessed using an electronic monitoring device (Smartinhaler@). Symptom control (Test for Respiratory and Asthma Control in Kids (TRACK)), quality of life (PACQLQ), airway inflammation (offline exhaled nitric oxide (FeNO)) were assessed at baseline and follow-up. Results: Forty-eight children (mean age 3.7 years, SD 1.2) were monitored for a median of 112 (IQR 91-126) days. At baseline $\mathrm{n}=29$ had episodic viral wheeze (EVW) and $\mathrm{n}=19$ had MTW. 24/48 (50\%) wheezers had sub-optimal ICS adherence $(<80 \%)$. Median adherence was $64 \%$ (IQR 38-84). There was a significant increase in TRACK in the group as a whole, unrelated to adherence. When split according to ICS responders, a significant increase in TRACK was only seen in atopic wheezers with adherence $>60 \%$. There was no relationship between clinical phenotype, adherence and symptom control. Conclusion: Adherence to ICS was sub-optimal and was positively related to symptom control in atopic wheezers only. Assessments of adherence are important in preschool children with an ICS responsive phenotype prior to therapy escalation.
\end{abstract}

\section{Introduction}

Wheezing in preschool children results in significant morbidity and healthcare costs worldwide[1-3]. Prevention of acute symptoms and hospitalisation is challenging because it is a heterogeneous disease, and little is known about the pathophysiological mechanisms underlying different phenotypes. However, there is good evidence that preschool wheezers with a phenotype that fits with "persistent asthma", characterised by recurrent and persistent wheezing, associated with a history of atopy in the child or immediate family, respond to maintenance inhaled corticosteroids (ICS) with a reduction in exacerbations and improved symptom control $[4,5]$. Children with a clinical phenotype of multiple trigger wheeze (wheeze during and between episodes) are also considered more steroid responsive than those with only episodic viral wheeze[6]. In addition, preschool wheezers with either or both of aeroallergen sensitisation and elevated blood eosinophils are thought to respond to ICS[7]. Although the efficacy of maintenance ICS has been assessed in specific phenotypes in the context of clinical trials, little is known about the relationship between adherence to prescribed ICS and efficacy in preschool children with troublesome wheeze in the clinic.

Adherence to maintenance ICS in school age children ranged from 20\%[8] up to 74\%[9], and higher adhe- 
rence was associated with better asthma control. In younger children aged (2-6 years) with wheeze/asthma, objectively monitored adherence for 12 months was reported as a median of $87 \%$ and $>80 \%$ adherence was associated with better symptom control assessed using the asthma control questionnaire[10]. In another study that included young children (18 months -7 years), median adherence rate was $71 \%$ with a range of 21-100\%[11]. Both studies that included young children used electronic monitoring devices (Smartinhalers) to assess adherence. However, neither reported the impact of adherence on symptom control in children with an objective phenotype that is likely responsive to ICS.

Much of the data that has shown relationships between objective monitoring of adherence and asthma control in school-age children has been in difficult asthma, the majority of such patients having allergic, eosinophilic disease which is steroid responsive. However, preschool wheezers may not all have an ICS responsive phenotype. Monitoring adherence to prescribed ICS may be beneficial in wheezers to help identify those with a steroid responsive phenotype. The impact of adherence to prescribed ICS in a population of preschool children with severe recurrent wheeze has not previously been reported. We hypothesised that firstly, adherence to prescribed ICS would be higher in children with severe preschool wheeze attending a specialist paediatric respiratory clinic compared to published data in school-age children with difficult asthma and secondly, that a period of adherence monitoring would result in improved symptom control only in those with ICS responsive features (multiple trigger wheeze or aeroallergen sensitisation). We investigated adherence to ICS using electronic monitoring devices for 4 months and related this to symptoms, offline exhaled nitric oxide (FeNO), quality of life and unscheduled healthcare visits in children aged 1-5 years with recurrent, troublesome preschool wheeze.

\section{Methods}

Design and setting

Prospective observational cohort study, with up to 4 months monitoring of children aged 1-5 years with recurrent, troublesome wheeze, who were seen regularly in the respiratory outpatient clinic at the Royal Brompton Hospital, London between March 2017 and November 2019. Children had been referred to our specialist respiratory clinic for further assessment or management of persistent and severe symptoms.

Ethical considerations

The study was approved by the Regional Ethical Committee (NRES Committee London-Westminster). All parents/legal guardians gave written informed consent.

Inclusion criteria and collection of baseline data

All eligible children had a documented diagnosis of preschool wheeze by a healthcare professional and were prescribed maintenance ICS by their clinician. Children with other significant primary pulmonary disorders were excluded. All children were classified into one of two groups at inclusion, based on pattern of symptoms. 1) Episodic viral wheeze (EVW): wheezing during discrete time periods, with absence of wheeze between episodes. 2) Multiple-trigger wheeze (MTW): wheezing in discrete exacerbations, but also symptoms between episodes[6].

Assessments were made at a baseline visit at the start of the monitoring period and at follow-up between 8-16 weeks later at the end of the monitoring period. All visits were undertaken as part of routine clinic visits, hence the variability in follow-up duration.

Symptom control

Symptom control was assessed using Test for Respiratory and Asthma Control in Kids (TRACK) questionnaire. This is a standardised tool validated to identify symptom control over the preceding 4 weeks in children with preschool wheeze[12]. It is a 5-item, 100-point caregiver-completed questionnaire, with poor asthma control defined as a score of $<80 / 100$ (see figure 1 online supplement). A change in score of $>10$ is the minimum clinically meaningful change[13]. 
Quality of life

All parents completed a parental and child quality of life questionnaire (PACQLQ)[14].

Exhaled nitric oxide

Exhaled nitric oxide (FeNO) was assessed using the offline technique using an electrochemical analyser (NIOX Vero; Aerocrine, Solna, Sweden) as previously described[15]. Published reference values for preschool healthy children were used to define the cut-off for normal (>11.5ppb)[16].

Atopic status

Atopy was defined as at least one positive skin prick test ( $>3 \mathrm{~mm}$ wheal) to one of a range of aero-allergens (house dust mite, grass, tree pollen, cat, dog).

Assessment of inhaler technique and adherence to ICS

All carers were shown how to use their child's inhaler with a spacer correctly. They were all given a written personalised wheeze plan with clear instructions about when the treatment should be administered. All families were issued with an electronic monitoring device at the baseline visit (Smartinhaler@; Nexus6 Ltd, Auckland, New Zealand). Smartinhalers were available for beclomethasone diproprionate, fluticasone proprionate and Seretide (fluticasone proprionate plus salmeterol combination inhaler) metered dose inhalers (MDIs). The devices contain a microchip that records the date and time the inhaler is actuated. Participants and their guardians were informed that the Smartinhaler would record the total number of actuations of ICS per day. Adherence was defined as the percentage of controller medication doses taken relative to the number of doses prescribed. Adherence was defined a priori as a ratio of doses taken; good adherence [?] $80 \%$, moderate adherence $60-79 \%$ and poor adherence $<60 \%[10]$. Daily adherence was calculated with a maximum of $100 \%$, to avoid falsely increased values due to dose dumping.

Follow-up

A minimum of 8 weeks and up to 16 weeks of adherence monitoring was undertaken. At follow up, symptom control using TRACK, PACQLQ and offline FeNO were assessed. In addition, unscheduled healthcare visits (UHCVs) for acute attacks were recorded.

\section{Analysis}

The associations between adherence and changes in PACQLQ, FeNO and symptom control from baseline to the end of the monitoring period were assessed using the non-parametric Wilcoxon Signed Rank Test, differences between unpaired data were assessed using the Mann Whitney test for 2 groups, or the Kruskal Wallis test for $>2$ groups. Data were analysed using Graphpad Prism v6 software and $\mathrm{p}<0.05$ was considered statistically significant.

\section{Results}

Forty-eight children mean age 3.7 (SD 1.2) years were included. Table 1 provides a summary of the baseline characteristics and prescribed treatments at inclusion. The median number of hospital admissions and daily dose of prescribed ICS met the criteria for severe, troublesome wheezing[17].

\section{Adherence to ICS and control of preschool wheeze symptoms}

Although 48 electronic monitoring devices were given to patients recruited, only 36/48 (75\%) were returned. This was despite repeated reminder phone calls from the nurse and despite waiting for up to 6 months for the device to be returned. There was no difference in the demographic characteristics at baseline between children whose monitors were and were not returned. Children were brought to the clinic for appointments, but families failed to return the monitoring device. The reported reasons for devices not being returned were loss of device or forgetting to return the device. 
Median period of monitoring for the 36 children who did return the monitoring device was 112 (range 91126 ) days. Median adherence during this period was $64 \%$ (range $0-94 \%$ ). $12 / 36(33 \%$ ) had $>80 \%$ adherence [good], 8/36 (22\%) had adherence between 60-79\% [moderate] and 16/30 (44\%) had <60\% adherence [poor]. There were no differences in baseline TRACK score, PAQLQ, FeNO or attacks in the preceding 3 months in those with good or suboptimal adherence $(<80 \%)$

There was a significant increase in TRACK score between baseline and follow-up visit for the children as a group, median TRACK at baseline 55 (Range 15-95), increasing to median TRACK at follow-up 70 (Range 25-95), p<0.01 (Fig 1A). However, there was no relationship between level of adherence and change in TRACK (Fig 1B). There was a significant improvement in PACQLQ between baseline and follow up visit for the group as a whole (Fig 1C), but no difference in offline FeNO measurements (Fig 1D) from baseline to follow-up.

The baseline and follow-up assessments were compared in children who had not returned the monitoring devices to those with sub-optimal $(<80 \%)$ and good $(>80 \%)$ recorded adherence. There was no difference in TRACK, PACQLQ, FeNO or unscheduled healthcare visits between the group who did not return the device and those with sub-optimal adherence. Comparing the children with recorded sub-optimal adherence and good adherence, only children with good adherence had a normal TRACK score (>80), significantly improved PACQLQ and a significantly lower number of unscheduled healthcare visits at follow-up (Table 2).

\section{Relationship between adherence and wheeze phenotype}

As episodic symptoms might have influenced whether parents gave maintenance treatment and episodic wheeze may be less responsive to ICS, we investigated the relationship between clinical phenotype and adherence. Sixty percent of children had episodic viral wheeze. Median adherence was similar in both phenotypes, EVW (63\% [0-91\%]) vs MTW (75\% [0-94\%]), p=ns.

\section{Relationship between adherence, atopic status and symptom control}

In order to determine whether change in symptom control with ICS treatment was related to objective biomarkers that determine steroid responsiveness, we assessed adherence and TRACK score at baseline and follow-up in atopic and non-atopic wheezers separately. Overall adherence was similar in atopic and nonatopic children (Table 3). There was a significant and clinically meaningful increase (TRACK increase of $>10$ ) in symptom score only in atopic wheezers from baseline to follow-up (Table 3 \& Fig 2A), while TRACK remained similar in non-atopic wheezers (Fig 2B). When all atopic wheezers were divided into those with moderate or good adherence $(>60 \%)$, compared to those with poor adherence $(<60 \%)$, only atopic wheezers with adherence $>60 \%$ had a significant increase in TRACK score from baseline to follow-up visits (Fig 3). This suggests symptom control and ICS adherence are only positively related in the sub-group of wheezers who have a steroid responsive phenotype.

\section{Discussion}

We investigated adherence to ICS in children aged 1-5 years with moderate to severe wheezing attending a tertiary respiratory centre. One-quarter of families did not return the electronic monitor, which meant an objective assessment of adherence could not be made, but suggests adherence was likely sub-optimal in that group. Of those who did return the monitor, adherence was sub-optimal $(<80 \%)$ in two-thirds.

Preschool children aged 2-6 years from a Dutch cohort with milder wheezing monitored for 12 months had median adherence of $87 \%[10]$. Only $33 \%$ of children in our study had good adherence $(>80 \%$ of prescribed doses), compared with $63 \%$ reported in the Dutch study. However, poor adherence to treatment may be rational, especially if the therapy prescribed is identified as ineffective by parents[18]. Hence parents of children with episodic viral wheeze, a group which may be less likely to be steroid responsive, may be right not to give the treatment. We therefore investigated the relationship between clinical phenotype, adherence and symptom control. However, there was no relationship between adherence to ICS and episodic viral or multiple trigger wheeze. In a randomised controlled trial (INFANT trial), children with aeroallergen sensitisation and an elevated peripheral blood eosinophil count responded best to ICS[7]. We did not have 
contemporaneous blood eosinophil counts in this study, but we were able to investigate whether atopic status influenced the relationship between adherence and response to ICS. Overall, only atopic wheezers had an improvement in TRACK score from baseline to follow-up. But importantly, only those atopic wheezers with> $60 \%$ adherence to ICS during the monitoring period had a significant and clinically meaningful improvement in symptom score. This suggests that this is indeed a steroid-sensitive group. In summary, even in those with troublesome wheezing, an improvement in symptom control is only apparent in atopic wheezers with moderate-good adherence to ICS.

There are other potential reasons for poor adherence, which we tried to exclude. We ensured the recruiting nurse provided education about the technique, need for the inhaler and a clear personalised wheeze plan. We had considered parental commitment to administering medication would be high in this group since most children had suffered frequent attacks requiring hospitalisation and the median dose of prescribed ICS was high[17]. A previous study in preschool children investigated reasons for poor adherence and found the most common reasons were parental forgetfulness and their child's reaction when the medication was given[11]. Administering inhaled therapy to children in this age-group is a challenge and lack of cooperation from the child may also be a reason for missed doses. Another contributory factor in this age group may be parental anxiety about the adverse effects of steroids[19].

Limitations of the study include the large number of families that did not return the monitoring device, but this was a pragmatic study undertaken in the clinic and this rate reflects our experience in school-age children[9], and highlights the potential costs incurred of lost devices (PS150 per device), especially if they are given indiscriminately to children unlikely to improve with ICS therapy. In addition, we did not assess change in TRACK score, or FeNO in children according to blood eosinophils as this had not been measured in the clinic. FeNO may have been influenced more by adherence to ICS in those with peripheral eosinophilia as is seen in older children and adults[20].

The implication of this and the previously published INFANT study[7] is that firstly, prescription of maintenance ICS should be limited to atopic preschool wheezers. We accept that when children have recurrent and severe symptoms, it is often easier to prescribe treatment, than do nothing, even if there is little evidence of efficacy. The ERS Task Force has stated (not on the basis of any evidence) that children with frequent and severe attacks may respond to ICS and so should be given a trial[21]. If this is to be done, the second implication of our study is that adherence needs to be assessed objectively. If ICS have been administered to the child, and there is no benefit, rather than escalating the dose, they should be discontinued.

We had hypothesised that adherence to ICS in this age group would be higher than in school-age children as parents would be committed to give the medication. However, adherence to ICS was sub-optimal in approximately half of children despite recurrent troublesome wheeze and being seen at a tertiary centre. Good adherence must not be assumed in this age group, and just as for older children with difficult asthma, if a preschool wheezer with a steroid responsive phenotype is not improving on high-dose ICS, then it is essential to ensure adequate adherence using objective monitoring prior to continued escalation of therapy. Getting the basics of management right is as important for preschool wheezers as for older children with asthma, especially as biologics may soon be considered for young children.

\section{Legends for Figures}

Figure 1. Change in symptom score, quality of life and exhaled nitric oxide between baseline and follow up visits. (A) Symptom score (TRACK) at baseline (Visit 1) and follow-up (Visit 2), (B) Change in TRACK according to level of monitored adherence, (C) Parental and child quality of life questionnaire (PACQLQ) and (D) exhaled nitric oxide (FeNO) at baseline and follow up.

Figure 2. Change in symptom score in atopic and non-atopic symptom between baseline and follow up visit. (A) Symptom score at baseline and follow-up in atopic children, (B) non-atopic children.

Figure 3. Change in symptom score in atopic children according to level of adherence. Relationship between adherence and change in symptom score from baseline (Visit 1) to follow up (Visit 2) in atopic children with 
(A) $>60 \%$ adherence to inhaled corticosteroids and (B) $<60 \%$ adherence to inhaled corticosteroids

Table 1. Baseline demographics of children included

\begin{tabular}{ll}
\hline $\mathbf{N = 4 8}$ & \\
\hline Age (years); mean (SD) & $3.7(1.2)$ \\
Male sex, n (\%) & $26(54 \%)$ \\
Atopy*, n (\%) & $25(52 \%)$ \\
TRACK score; median (range) & $55(15-95)$ \\
PACQLQ score; median (range) & $5.5(1.0-7.0)$ \\
Admissions for wheeze ever; median (range) & $3(0-20)$ \\
Offline FeNO (ppb); median (range) & $14(5-95)$ \\
ICS dose/day (BDP); median mcg (range) & $400(100-1000)$ \\
Clinical phenotype: EVW; n & $29 / 48(60 \%)$ \\
\hline
\end{tabular}

*Atopy defined as $>1$ positive skin prick test, or $>1$ positive specific $\operatorname{IgE}$ to aero-allergen $(>3 \mathrm{~mm}$ wheal diameter), ICS: inhaled corticosteroids, BDP: beclomethasone diproprionate, TRACK: test for respiratory and asthma control in kids, PACQLQ: parental asthma child quality of life questionnaire, FeNO: exhaled nitric oxide

Table 2. Relationship between level of adherence and control of preschool wheeze at beginning and end of monitoring

\begin{tabular}{lll}
\hline $\mathbf{N = 4 8}$ & Level of adherence & Level of adherence \\
\hline & Suboptimal $\mathbf{n = 2 4}$ & Good $\mathbf{n = 1 2}$ \\
TRACK score baseline & $55(15-85)$ & $45(30-85)$ \\
TRACK score end & $65(25-95)$ & $85(40-95)$ \\
$\mathrm{P}^{*}$ & $\mathbf{0 . 0 2 5}$ & $\mathbf{0 . 0 0 3 9}$ \\
PACQLQ baseline & $6.2(1.0-7.0)$ & $5.6(3.8-7.0)$ \\
PACQLQ score end & $6.8(2.8-7.0)$ & $6.8(3.8-7.0)$ \\
$\mathrm{P}^{*}$ & 0.3832 & $\mathbf{0 . 0 2 3 4}$ \\
Offline FeNO baseline $(\mathrm{ppb})$ & $24(5-94) \mathrm{N}=12$ & $8.5(5-95) \mathrm{N}=7$ \\
Offline FeNO end $(\mathrm{ppb})$ & $7.5(5-23)$ & $7.0(5.0-50.0)$ \\
$\mathrm{P}^{*}$ & 0.0977 & 0.8125 \\
UHCVs & $1(0-4)$ & $0(0-1)$ \\
$\mathrm{P}^{*}$ & $\mathrm{~N} / \mathrm{A}$ & $\mathrm{N} / \mathrm{A}$ \\
\hline
\end{tabular}

TRACK: test for respiratory and asthma control in kids, PACQLQ: parental asthma child quality of life questionnaire, FeNO: exhaled nitric oxide, ppb: parts per billion; UHCV: unscheduled healthcare visits

Table 3. Relationship between atopic status, adherence and wheeze control

\begin{tabular}{llll}
\hline $\mathbf{N = 4 8}$ & Atopy & Atopy & $\mathbf{P}^{* *}$ \\
\hline & Atopic $(\mathbf{n}=\mathbf{2 4})$ & Non-atopic $(\mathbf{n = 2 4 )}$ & \\
Adherence & $52.5(0-91)$ & $76(0-94)$ & 0.0642 \\
TRACK score baseline & $50(15-85)$ & $65(20-85)$ & 0.1095 \\
TRACK score follow-up & $72.5(30-95)$ & $67.5(25-95)$ & 0.5428 \\
P* & $\mathbf{0 . 0 0 1 8}$ & $\mathbf{0 . 3 7 7 9}$ & \\
PACQLQ baseline & $5.15(1.0-7.0)$ & $5.65(1.0-7.0)$ & 0.377 \\
PACQLQ follow-up & $6.8(2.8-7.0)$ & $6.65(1.0-7.0)$ & 0.7414
\end{tabular}




\begin{tabular}{llll}
\hline $\mathbf{N = 4 8}$ & Atopy & Atopy & $\mathbf{P}^{* *}$ \\
\hline $\mathrm{P}^{*}$ & 0.2975 & 0.0803 & \\
Offline FeNO baseline (ppb) & $24(5-94)$ & $8.5(5-95)$ & 0.4575 \\
Offline FeNO follow-up (ppb) & $6(5-30)$ & $8(5-50)$ & 0.832 \\
$\mathrm{P}^{*}$ & 0.1953 & 0.8438 & \\
\hline
\end{tabular}

\section{References}

1. Laforest, L., et al., Association between asthma control in children and loss of workdays by caregivers. Ann Allergy Asthma Immunol, 2004. 93 (3): p. 265-71.

2. Sritipsukho, P., et al., Economic Burden of Hospitalization with Acute Wheezing in Preschool Children: A Multi-Center Study. J Med Assoc Thai, 2016. 99 Suppl 4 : p. S239-45.

3. Stevens, C.A., et al., The economic impact of preschool asthma and wheeze. Eur Respir J, 2003.21 (6): p. $1000-6$.

4. Kaiser, S.V., et al., Preventing Exacerbations in Preschoolers With Recurrent Wheeze: A Meta-analysis. Pediatrics, 2016.137 (6).

5. Castro-Rodriguez, J.A. and G.J. Rodrigo, Efficacy of inhaled corticosteroids in infants and preschoolers with recurrent wheezing and asthma: a systematic review with meta-analysis. Pediatrics, 2009.123 (3): p. e519-25.

6. Brand, P.L., et al., Definition, assessment and treatment of wheezing disorders in preschool children: an evidence-based approach.Eur Respir J, 2008. 32 (4): p. 1096-110.

7. Fitzpatrick, A.M., et al., Individualized therapy for persistent asthma in young children. J Allergy Clin Immunol, 2016.138 (6): p. 1608-1618 e12.

8. McNally, K.A., et al., Adherence to combined montelukast and fluticasone treatment in economically disadvantaged african american youth with asthma. J Asthma, 2009. 46 (9): p. 921-7.

9. Jochmann, A., et al., Electronic monitoring of adherence to inhaled corticosteroids: an essential tool in identifying severe asthma in children. Eur Respir J, 2017. 50 (6).

10. Klok, T., et al., It's the adherence, stupid (that determines asthma control in preschool children)! Eur Respir J, 2014.43 (3): p. 783-91.

11. Burgess, S.W., et al., Assessing adherence and factors associated with adherence in young children with asthma. Respirology, 2008. 13 (4): p. 559-63.

12. Murphy, K.R., et al., Test for respiratory and asthma control in kids (TRACK): a caregiver-completed questionnaire for preschool-aged children. J Allergy Clin Immunol, 2009. 123 (4): p. 833-9 e9.

13. Zeiger, R.S., et al., Test for Respiratory and Asthma Control in Kids (TRACK): clinically meaningful changes in score. J Allergy Clin Immunol, 2011. 128 (5): p. 983-8.

14. Juniper, E.F., et al., Measuring quality of life in children with asthma. Qual Life Res, 1996. 5 (1): p. $35-46$.

15. Singer, F., et al., Exhaled nitric oxide in symptomatic children at preschool age predicts later asthma. Allergy, 2013.68 (4): p. 531-8.

16. van der Heijden, H.H., et al., Reference values of exhaled nitric oxide in healthy children 1-5 years using off-line tidal breathing. Pediatr Pulmonol, 2014. 49 (3): p. 291-5.

17. Global Strategy for Asthma Management and Prevention . 2020, Global Initiative for Asthma: Available from www.ginasthma.org. 
18. Yoos, H.L., H. Kitzman, and A. McMullen, Barriers to anti-inflammatory medication use in childhood asthma. Ambul Pediatr, 2003. 3 (4): p. 181-90.

19. Ozceker, D., et al., Corticosteroid phobia among parents of asthmatic children. Turk J Pediatr, 2018. 60 (2): p. 142-146.

20. Fricker, M., L.G. Heaney, and J.W. Upham, Can biomarkers help us hit targets in difficult-to-treat asthma? Respirology, 2017.22 (3): p. 430-442.

21. Brand, P.L., et al., Classification and pharmacological treatment of preschool wheezing: changes since 2008. Eur Respir J, 2014. 43 (4): p. 1172-7.

Figure 1.

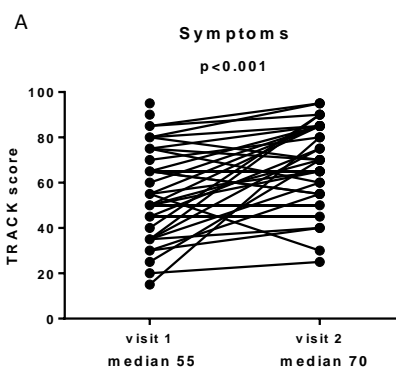

B

C

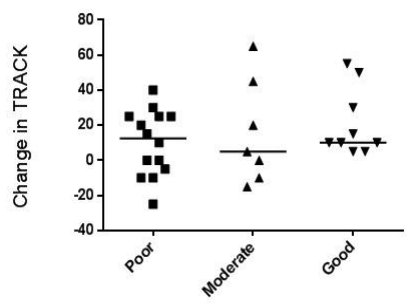

D
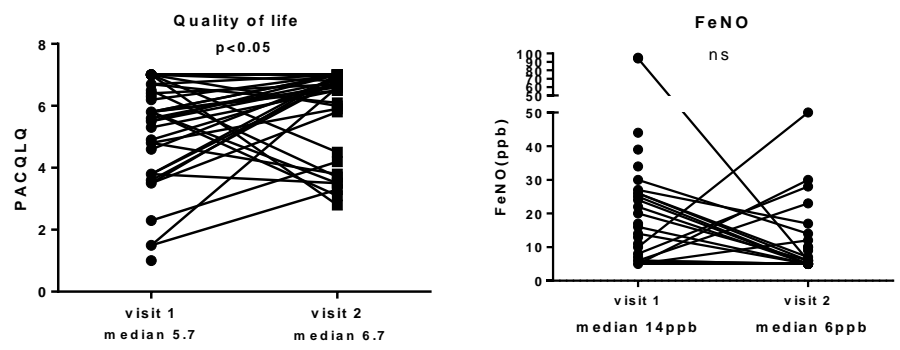
Figure 2.

A

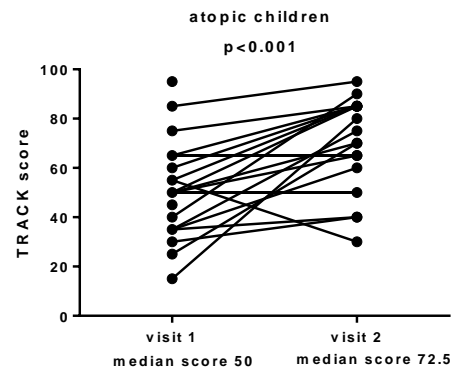

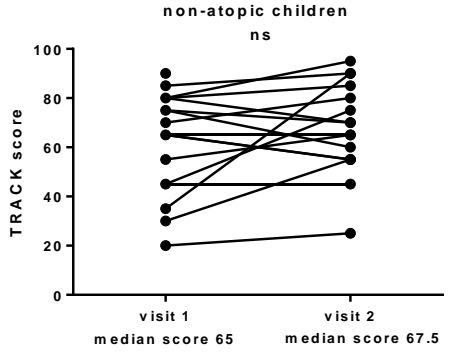


Figure 3.
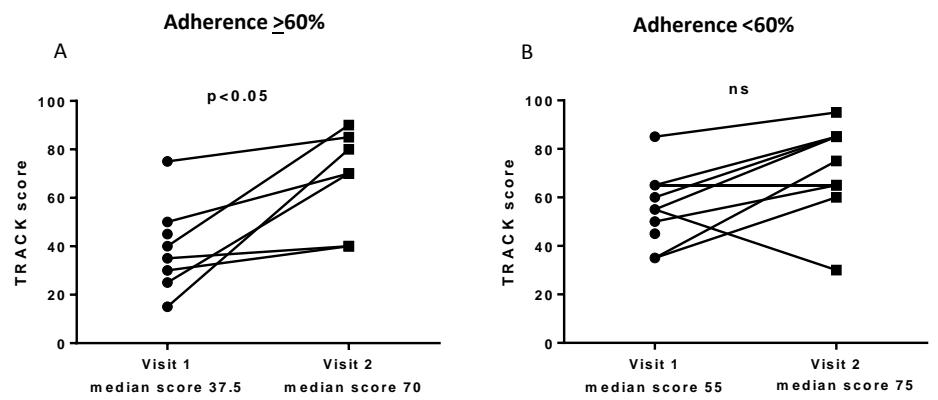


\section{Supplementary Figure 1}

Test for Respiratory and Asthma Control in Kids (TRACK) score questionnaire
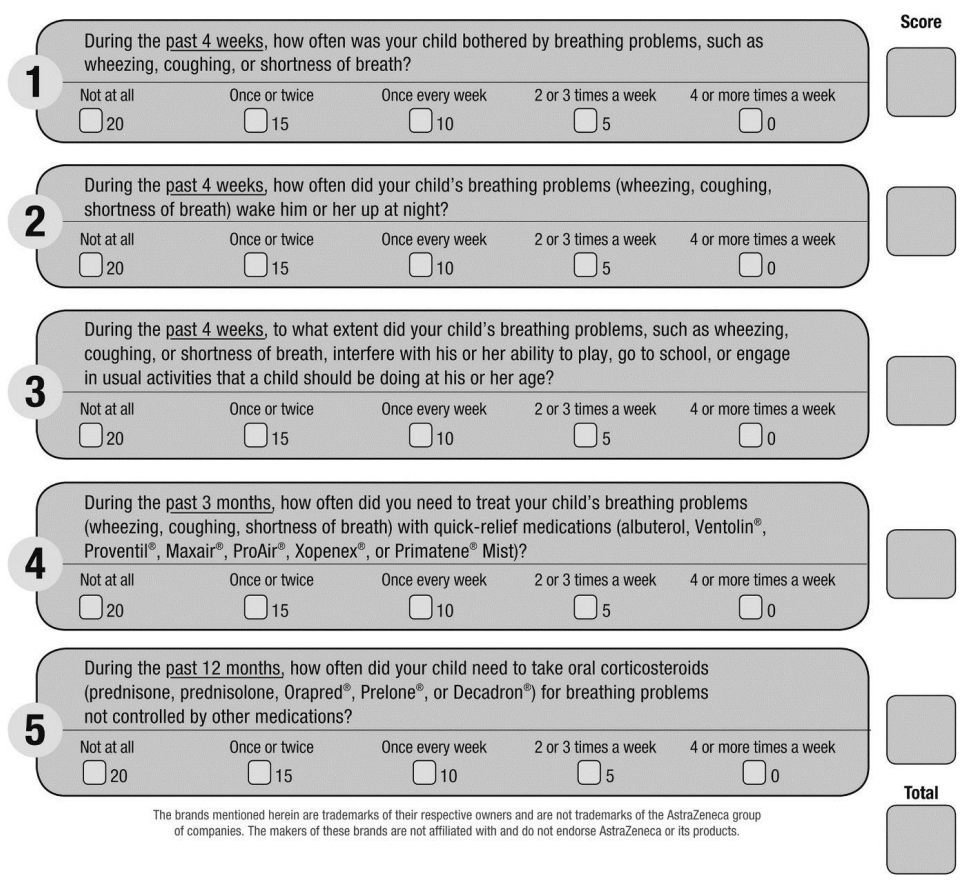

Adapted from: Zeiger RS et al J Allergy Clin Immunol 2011;128:983-988 